\title{
Ammartiamo? Meglio di no.
}

\author{
Enzo Caffarelli
}

PUBBLICATO: 02 APRILE 2019

\section{Quesito:}

Sollecitati dalle molte richieste proponiamo una riflessione sull'uso dei termini ammartare e ammartaggio.

\section{Ammartiamo? Meglio di no.}

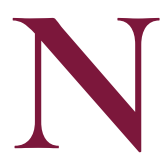

umerosi lettori hanno chiesto un parere sull'uso giornalistico dei termini ammartare e ammartaggio, ricorsi in occasione dell'importante evento astronomico in cui il suolo di Marte è stato toccato dalla sonda americana InSight, il 26 novembre 20I8. Ecco un paio di esempi nei titoli apparsi il giorno stesso dell'avvenimento: "Insight su Marte: ammartaggio perfetto, aperti i pannelli solari" (Matteo Marini, Insight su Marte: ammartaggio perfetto, aperti i pannelli solari, la Repubblica.it, Scienze); "L'ammartaggio della sonda InSight in diretta" (L'ammartaggio della sonda InSight in diretta, Focus.it).

Non si tratta di un neologismo dell'ultimo momento, beninteso; la prima attestazione nota ha oltre 40 anni. Il Vocabolario Treccani, consultabile in rete, lemmatizza ammartaggio come neologismo del 2012, ma ne rileva un'attestazione di Adriano Buzzati Traverso già nel quotidiano "La Stampa" del 23 luglio I976; lo stesso dizionario segnala come neologismo del 2014 ammartare, anche in questo caso retrodatandolo al XX secolo (Gabriele Beccaria, "La Stampa", i3 luglio i997) e chiosandolo come "Derivato dal nome del quarto pianeta del sistema solare, Marte, con l'aggiunta del prefisso ad-e del suffisso -are ${ }^{\mathrm{I}}$, sul modello di atterrare, ammarare, allunare e simili".

Vale la pena di segnalare quanto scrive Roberto Todini nell'articolo La Sonda InSight è Ammartata Sana e Salva, ancora il 28 novembre 20I8, su Ultima voce.it: «leri poco prima delle 2r ora italiana la sonda InSight è ammartata (il neologismo ammartare potrà suonare curioso ma a parer mio è sempre meglio della contraddizione in termini "atterrare su Marte") [...]». Tale opinione è evidentemente condivisa da almeno una parte degli operatori dell'informazione.

Pare tuttavia che la contraddizione sia invece proprio nelle voci ammartare e derivati. Il ricorso che se ne fa, come del resto segnalato da alcuni nostri lettori che non lo condividono, non tiene infatti conto dei due significati prevalenti di terra, che non sono il nome proprio del pianeta, ma a) il nome comune per indicare 'suolo', 'superficie solida' in opposizione a quella dei bacini acquiferi e di superfici liquide in genere, 'terraferma', e b) il valore di 'suoli e terreni formati da rocce appartenenti a varie categorie geotecniche, materiale costitutivi del terreno contenenti gli elementi necessari per la nutrizione delle piante'. È evidente che espressioni come cadere a terra, sedere a terra, andare a terra, gettare a terra, respingere una palla da terra, scavare sottoterra, ecc. non indicano il pianeta; e terra vale 'terreno coltivabile' in arare / fertilizzare / zappare la terra, vale 'materiale particolare' in vaso di terra, 'appezzamento di terreno' in ettaro di terra, 'zona geografica, territorio', in terra di Romagna.

Certo, ha pieno senso usare ammarare, ammaraggio in opposizione ad atterrare / atterraggio, ma parliamo sempre del nostro pianeta. Ben lungi dall'aprire la strada al pur frequente allunare / allunaggio (voci registrate per esempio dal GRADIT di Tullio De Mauro e datate entrambe al I959), 
l'allusione alle acque terrestri si colloca nellopposizione terra / mare che nulla ha a che fare con l'opposizione Terra / Luna: tanto che pare congruo parlare di atterraggio e ammaraggio sulla luna (anche se il secondo è solo ipotetico: i mari lunari, com’è noto, corrispondono a varie configurazioni morfologiche prive di acque).

D'altra parte, se per esempio un idrovolante o un uccello si posa sulle acque di un fiume o di un lago, non si ricorre a formazioni parasintetiche con base fiume o lago. In questo caso è assai curioso quanto si legge nella pagina ammaraggio di Wikipedia, da cui si dedurrebbe che ammaraggio non è che un iponimo di atterraggio: "Un ammaraggio è, in senso ampio, qualunque atterraggio su uno specchio d'acqua [...]. Gli idrovolanti, siano essi a scafo, a galleggianti o anfibi, sono progettati per decollare e atterrare sull'acqua"; e più avanti, nel paragrafo "Ammaraggio d'emergenza", si legge "[...] il comandante ha dovuto prendere la difficile decisione di eseguire un atterraggio di emergenza sul fiume" (corsivi aggiunti).

Se ne può concludere che atterrare su $X$ con $X$ satellite o pianeta, nel senso di 'toccare il suolo, poggiare sulla superficie nella fattispecie della Luna o di Marte' è espressione pienamente corretta, mentre ammartare / ammartaggio è un semplice occasionalismo, il cui uso in una circostanza particolare come quella indicata all'inizio si può certamente capire, ma che non è destinato a inserirsi stabilmente nel lessico, perché presupporrebbe per coerenza l'uso dell'astronimo in ogni situazione e azione (al momento perlopiù non plausibile) in cui, sul nostro pianeta, ci trovassimo a nominare terra (*cadere a Marte, *sedere a Marte, " "gettare a Marte...). Per non dire della catena di parasintetici cacofonici da tenere in caldo per le future conquiste spaziali: avveneraggio, ammercurare, aggiovaggio, assaturnato... fino ad applutonato.

\section{Cita come:}

Enzo Caffarelli, Ammartiamo? Meglio di no. , "Italiano digitale", 2019, IX, 2019/2 (aprile-giugno) DOI: 10.35948/2532-9006/2020.3096 